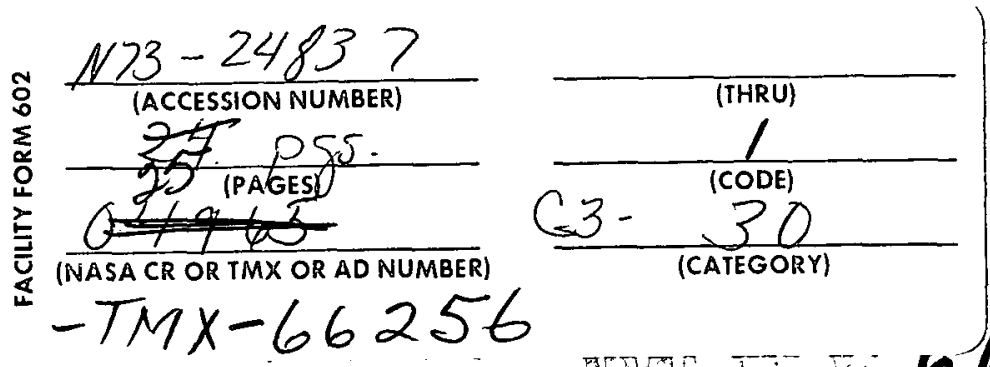

$X-646-73-163$

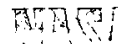

\title{
RATE OF EROSION OF
}

DAYSIDE MAGNETIC FLUX BASED ON A QUANTITATIVE STUDY OF THE DEPENDENCE OF POLAR CUSP LATITUDE ON THE INTERPLANETARY MAGNETIC FIELD

\section{J. L. BURCH}

\section{JUNE 1973}

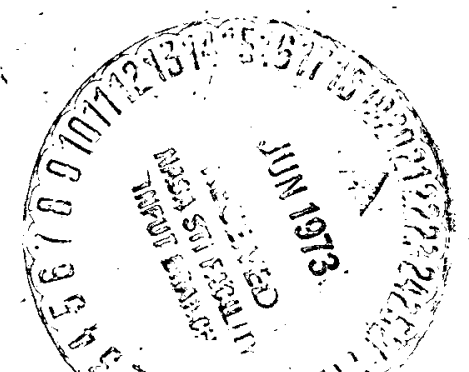

GSFC - GODDARD SPACE FLIGHT CENTER GREENBELT, MARYLAND 
RATE OF EROSION OF DAYSIDE MAGNETIC FLUX

BASED ON A QUANTITATIVE STUDY OF THE DEPENDENCE OF POLAR CUSP

LATITUDE ON THE INTERPLANETARY MAGNETIC FIELD

\author{
By \\ J. L. Burch \\ Laboratory for Space Physics \\ NASA-Goddard Space Flight Center \\ Greenbe1t, Maryland 20771
}

Paper presented at the Chapman Memorial Symposium on Magnetospheric Motions, Boulder, Colorado, June 18-22, 1973

I 


\section{ABSTRACT}

By considering only those periods when the delay time from the interplanetary observing position to the magnetosphere is less than $\sim 5$ minutes, it is found that, irrespective of substorm activity: (1) The 45-minute average value of interplanetary $B_{Z}$ predicts the latitudes of the poleward and equatorward boundaries of polar cusp electron precipitation with rms errors of $1.34^{\circ}$ and $1.16^{\circ}$ respectively; (2) Both boundaries move equatorward by about $5^{\circ}$ as $\mathrm{B}_{\mathrm{Z}}$ varies from 0 to $-6 \%$, the cusp remaining about $4^{\circ}$ wide; (3) The amount of flux added to the polar cap is about $9.2 \%$ of the total southward flux impingent on the magnetosphere in the previous 45-minutes; (4) As $B_{Z}$ becomes more positive, the equatorward boundary moves only slightly more poleward $\left(\frac{1}{2}^{\circ}\right.$ between $B_{Z}=2 \gamma$ and $B_{Z}=6 \gamma$ ), while the poleward boundary moves significantly toward higher latitudes, resulting in a cusp approximately $7^{\circ}$ wide for $B_{Z}=6 \gamma$. This dependence of the width of the cusp on positive $B_{Z}$ suggests that reconnection between the IMF and open geomagnetic field lines (which are southward near the dayside magnetopause) is facilitated when the IMF is northward, allowing deeper penetration of magnetosheath plasma into the high-latitude magnetosphere.

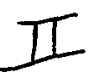




\section{INTRODUCTION}

The inward motion of the magnetopause, and the associated equatorward motion of the polar, or dayside, cusps during periods of substorm activity has been well-established by Meng (1970) and Akasofu (1972). However, the close association between southward interplanetary magnetic fields (IMF) and substorm activity has made difficult the determination of which of these phenomena actually causes the polar cusp equatorward movement. An observation of the actual inward movement of the magnetopause following a southward-turning of the IMF and before substorm onset by Aubry et a1 (1970) suggested that the inward movement was caused by erosion of dayside magnetic flux associated with the southward IMF. Observation of successive poleward and equatorward movement of the highaltitude polar cusp in response to southward and northward IMF transitions by Russe11 et a1 (1971) during the magnetic storm of November 1, 1968, a1so agrees wel1 with this concept.

An earlier communication (Burch, 1972) reported that the equatorward boundary of polar cusp electron precipitation was found at abnormally low latitudes after periods of southward IMF even when very little substorm activity had occurred, indicating again that the southward IMF is the chief cause of polar cusp equatorward movement. By further relating this latitude to the period of time between sharp transitions to southward IMF and the polar cusp observations, a gradual equatorward movement of approximate1y $0.1 \%$ min. was inferred for periods of sustained southward IMF of less than $1 \frac{1}{2} \mathrm{hrs.}$. and average solar-magnetospheric (GSM) southward components less than $7 \gamma$. 
For the ten well-defined north-south transitions examined in the previous study, ratios of 0.05 to 0.30 were found between the amount of southward magnetic flux brought to the front of the magnetosphere and the dayside magnetospheric flux eroded into the tail or polar caps. Measurements from low-altitude satellites are well-suited for the determination of such variations of total dayside magnetic flux, including that due to boundary currents, since, by flux conservation, the dipole magnetic flux crossing the earth's surface on closed field lines must equal the total flux crossing the equatorial plane. Therefore, while it is difficult to relate a movement of the equatorial magnetopause to a quantitative change in dayside magnetic flux, it is easy to do so if the latitude of the last closed field line is known at low altitudes. Unfortunately, sharp transitions to a sustained southward IMF do not occur frequently. Therefore, if a method of predicting the polar cusp latitude is sought, it must include effects of complicated IMF changes as we11. The purpose of this study is to investigate the accuracy with which the northern-hemisphere polar cusp latitude can be predicted based only upon the average value, over an appropriate time scale, of a single parameter of the interplanetary field. The quantitative relationship obtained is then used to refine previous estimates (Burch, 1972) of the efficiency of erosion of dayside magnetic flux by southward interplanetary magnetic fields.

\section{POLAR CUSP MEASUREMENTS}

Electron data from the OGO-4 Auroral Particles Experiment (Hoffman and Evans, 1968) are used in this study. The experiment consisted of seven cylindrical electrostatic analyzers measuring directional fluxes 
of $0.7,2.3,7.3$ and $23.9 \mathrm{kev}$ electrons arriving along the earth's radius vector and fluxes of $2.3 \mathrm{kev}$ electrons at $30^{\circ}, 60^{\circ}$ and $90^{\circ}$ to the radius vector. In high-latitude regions, where $\bar{B}$ is nearly radial at the 0G0-4 altitudes of 400 to $900 \mathrm{~km}$, the $0^{\circ}$ detectors measured precipitated electrons.

A typical example of the electron fluxes measured in the high-latitude soft zone, or polar cusp, is shown in Figure 1. In this study we restrict attention to the $9-15 \mathrm{hr}$ MLT sector where a distinct equatorward boundary of polar cusp electron precipitation is easily discernible in every high-latitude pass of 0G0-4. The lower-latitude, more diffuse, hard zone, consisting of drifting substorm-injected electrons of much higher energies than the polar cusp, or soft zone, electrons, is present only after a period of substorm activity (Hoffman and Burch, 1973). As shown in Figure 1, a distinct poleward boundary, separating the polar cusp electrons from the polar cap, is often apparent. However, unambiguous identification of this poleward boundary is impossible in many cases due to the isolated spikes or drizzle of precipitation into the polar cap such as reported by Heikkila and Winningham (1972). This results in a smaller number of poleward boundaries than equatorward boundaries available for study. A1though, as suggested by the work of Kivelson et a1 (1973), the IMF direction may have important effects on the electron distribution functions in the low-altitude polar cusp, the fact that the 0G0-4 experiment could only measure the high-energy tail of the electron spectrum made it unsuitable for that type of investigation. However, the OG0-4 measurements do provide reliable determinations of the location of the region 
of polar cusp electron precipitation. In this study we interpret the equatorward boundary of precipitation as defining the position of the last magnetic field line which closes on the dayside, and the poleward boundary as defining the highest-latitude field line to which magnetosheath electrons have direct access. The intervening field 1 ines are a11 presumably connected directly to the magnetosheath magnetic field, and so are being swept back into the downstream magnetosheath and magnetotail.

INTERPLANETARY MAGNETIC FIELD MEASUREMENTS

If the interplanetary magnetic field is to be related quantitatively to the instantaneous position of the polar cusp, it is necessary to determine the delay time between the interplanetary observing position and the front of the magnetosphere with an error small with respect to the time scale of polar cusp movement. Large errors can result when measurements are made very far off the earth-sun line, especially when the IMF azimuth is near $0^{\circ}$ or $180^{\circ}$. Also, unacceptable errors can result if measurements at large radial distances are used, especially if simultaneous solar-wind velocity measurements are not available.

During the periods, late July to early October, 1967 and 1968, the orbit of Explorer 34 was suitable for obtaining IMF measurements near the earth-sun line at radial distances less than $35 \mathrm{R}_{\mathrm{E}}$, and a large number of polar cusp observations were made by OGO-4. The delay time from $35 \mathrm{R}_{\mathrm{E}}$ to a typical magnetopause at $11 \mathrm{R}_{\mathrm{E}}$ is $\sim 6$ minutes for a solar wind velocity of $450 \mathrm{~km} / \mathrm{sec}$. Therefore, even when solar-wind velocity measurements were not available, errors in the determination 
of the delay times were less than 5 minutes. By considering only periods when Explorer $34\left|\mathrm{Y}_{\mathrm{GSM}}\right|$ components were less than $12 \mathrm{R}_{\mathrm{E}}$, delay time errors were maintained at less than $\sim 5$ minutes even when the IMF azimuth was near $0^{\circ}$ or $180^{\circ}$. The inclination of the Explorer 34 orbit itself restricted Explorer $34\left|\mathrm{Z}_{\mathrm{GSM}}\right|$ components to less than $13 \mathrm{R}_{\mathrm{E}}$.

These delay time considerations were the only constraints used in selecting the polar cusp boundary measurements for analysis. These constraints resulted in 54 equatorward polar cusp boundaries and 21 poleward boundaries. Explorer $34 \mathrm{X}, \mathrm{Y}_{\mathrm{GSM}}$ locations for these 75 cases are plotted in Figure 2 along with the average bow shock and magnetopause taken from Fairfield (1971).

DEPENDENCE OF POIAR CUSP LATITUDE ON THE INTERPLANETARY MAGNETIC FIELD

Having previously determined (Burch, 1972) that a southward IMF is the chief cause of polar cusp equatorward displacement and that a systematic gradual movement occurs after sharp transitions to sustained southward fields, we now investigate whether it is possible to predict the positions of the polar cusp equatorward and poleward boundaries for an arbitrarily-varying field. Coroniti and Kennel (1971) have suggested that the process of erosion of dayside magnetic flux into the tail and its subsequent return by convection is continually approaching an equilibrium state. According to the Coroniti and Kennel mode1, magnetospheric convection grows and decays exponentially, due to ionospheric line-tying, with a time constant $\Delta$ which they estimated at approximately 20 minutes. In such a model, a time of order $2 \Delta$ would be required for convection and the polar cusp location to approach their equilibrium 
states for a condition of constant $\bar{B}_{\text {IMF }}$ and solar wind velocity. The Coroniti and Kennel model further assumes no erosion of dayside magnetic flux for northward IMF's and an erosion rate for southward IMF's that is proportiona1 to the southward component.

One would like to know, in the context of such a model, whether or not knowledge of the average IMF southward component over a given period of time before a polar cusp observation could be used to predict its latitude. An appropriate time scale for such an average would be approximately $2 \Delta$, since earlier IMF variations would largely be "forgotten" by the magnetosphere. If all magnetic flux at latitudes above that of the equatorward polar cusp boundary is termed polar cap flux, its rate of change should be expressible as $\dot{\Phi}_{P C}=\dot{\Phi}_{0}-\dot{\Phi}_{R}$, where $\dot{\Phi}_{0}$ is the rate at which flux is added to the polar cap and $\dot{\Phi}_{R}$ is the rate at which flux is returned to the dayside by convection (Coroniti and Kennel, 1971). With an exponential growth and decay of convection, which is driven by flux erosion and limited by ionospheric Pederson currents, one has $\dot{\Phi}_{\mathrm{R}}=\dot{\Phi}_{0}\left[1-\exp \left(-t_{\text {south }} / \Delta\right)\right]$ for a southward IMF, and, after a subsequent northward transition, $\dot{\Phi}_{R}=\dot{\Phi}_{0}\left[1-\exp \left(-t_{\text {south }} / \Delta\right)\right] \exp \left(-t_{\text {north }} / \Delta\right)$.

the doove relacionsnips predict that atter equilibrium is reached, with a constant southward IMF, an amount of flux equal to $\dot{\Phi}_{0} \Delta$ will have been added to the polar cap. In Figure 3 are shown schematically what one would expect for three different types of IMF variation, a11 of which result in the same average southward component $\left(B_{O}\right)$ over a time interval $2 \Delta$ before a hypothesized polar cusp observation (at $t=t_{0}+$ $2 \Delta)$. The solar-wind velocity $\left(v_{S}\right)$ is assumed constant for all three 
cases, since $\dot{\Phi}_{0}$ is expected to depend on both $B_{-Z}$ and $v_{S}$. In case number 1 of Figure 3 , the IMF turns southward at time $t_{0}-2 \Delta$, and maintains the value $B_{Z}=-B_{0}$ until the polar cusp observation, at which time $\Phi_{P C}-\Phi_{P C}(0)$ (or the flux added to the polar cap) approaches very closely the equilibrium value $\dot{\Phi}_{0} \Delta$. In case number 2 , the IMF also switches southward at time $t_{0}-2 \Delta$, but to a value $B_{Z}=-2 B_{0}$, or twice as large as in case 1 . At time $t_{0}+\Delta$ a transition to an arbitrary positive $B_{Z}$ occurs. In this case, the additional flux in the polar cap at the time of the polar cusp observation is .71 $\dot{\Phi}_{0} \Delta$. The southward transition occurs later in case number 3 , at time $t_{0}+\Delta$. The transition is again to a level $B_{Z}=-2 B_{0}$, and at the time of polar cusp observation $\left(t_{0}+2 \Delta\right)$ the additional flux in the polar cap is equal to $1.26 \dot{\Phi}_{0} \Delta$. Although in actuality more complicated variations occur, the cases depicted in Figure 3 suggest that errors should be not much greater than $30 \%$, and that knowledge of the average IMF southward component may be expected to provide a fairly reliable estimate of the location of the polar cusp.

As mentioned above, the only criterion used in selecting 0G0-4 polar cusp latitude determinations for analysis was the availability of suitable Explorer 34 IMF measurements near the earth-sun line. Substorm or magnetic storm activity was purposely not considered since it is hypothesized, based on previous work (Burch, 1972), that effects due to phenomena other than the southward IMF are of secondary importance.

Although it was found previously (Burch, 1972) that there is a $4^{\circ}$ yearly variation in the latitude of the cusp equatorward boundary due to dipole tilt, the constraints on the interplanetary observing location 
in fact limited this analysis to dipole-axis - earth-sun-line angles between $75^{\circ}$ and $105^{\circ}$, for which only a $2^{\circ}$ variation in cusp latitude is expected.

For each of 54 OGO-4 crossings of the cusp equatorward boundary, average values of the IMF $B_{Z}$ and $\theta$ were computed for periods of 15-, 30-, 45- and 60-minutes. For the 28 cases when solar-wind velocity measurements were available from Explorers 33,34 or 35 , the Explorer 34 IMF measurements were corrected in time to an assumed magnetopause at $\mathrm{X}_{\mathrm{GSM}}=$ $10 R_{E}$. When velocity measurements were not available, a typical velocity of $450 \mathrm{~km} / \mathrm{s}$ was used for this purpose. It was found that the cusp latitudes were ordered much better using $B_{Z}$ than $\theta$. The results for the 45-minute averages of $B_{Z}$ are shown in Figure 4 (solid points). The solid curve is a least-squares quadratic fit to the data, for which the root mean square deviation is $1.16^{\circ}$. The other three time periods used resulted in similar curves. However, the root mean square deviations for the 15-, 30-, and 60 - minute averages were $1.27^{\circ}, 1.18^{\circ}$, and $1.20^{\circ}$ respectively. In light of the discussion of Figure 3 , these results indicate that 45 minutes is a somewhat better estimate for $2 \Delta$ than the other times considered. This is an agreement with the estimate of 40 minutes made by Coroniti and kenme $1+y / 1)$.

As shown by the lower curve of Figure 4, a systematic lowering of the cusp equatorward boundary of $\sim 5^{\circ}$ occurs between $B_{Z}=0$ and $B_{Z}=$ - 6y. In contrast, this boundary moves poleward by only about $1 \frac{1}{2}^{\circ}$ for positive values of $B_{Z}$ up to $+6 \gamma$, with only about a $\frac{1}{2} \mathrm{O}$ change between $+2 \gamma$ and $+6 \gamma$. This result supports the hypothesis of Coroniti and Kennel (1971) that the flux in the polar cap is indpendent of $B_{Z}$ for a northward 
IMF. As indicated in Figure 4, the equation $\Lambda=75.5^{\circ}+0.53 \mathrm{~B}_{\mathrm{Z}}-0.05 \mathrm{~B}_{\mathrm{Z}}{ }^{2}$, with $B_{Z}$ in gammas, predicts the latitude of the cusp equatorward boundary to an accuracy of better than $3^{\circ}$ for $-6 \gamma \leq B_{Z} \leq 6 \gamma$.

The results of a similar analysis for the poleward boundary of dayside cusp electron precipitation are shown in the upper plot of Figure 4. The quadratic fit to these points $\left(\Lambda=80.3^{\circ}+0.76 \mathrm{~B}_{Z}-0.03 \mathrm{~B}_{Z}{ }^{2}\right)$ is nearly parallel to that for the equatorward boundary for negative $B_{Z}$ (southward IMF), indicating a cusp approximately $4^{\circ}$ wide. However, in contrast with the behavior of the equatorward boundary, the poleward boundary moves significantly to higher latitudes as $B_{Z}$ becomes more positive, resulting in a cusp approximately $7^{\circ}$ wide for IMF northward components approaching $6 \gamma$. The root mean square deviation for this curve is $1.34^{\circ}$.

The cusp behavior shown in Figure 4 may be interpreted as shown schematically in Figure 5(a), (b) and (c). In Figure 5(a) the IMF is nearly horizontal and a minimum of reconnection occurs. The dayside closed magnetic field lines are generally directed northward at the magnetopause, while open field lines, which are swept back into the tail, are southward at the dayside magnetopause. In Figure $5(\mathrm{~b})$ the onset of southward IMF facilitates reconnection with closed field lines and subsequent erosion of dayside flux into the polar cap. As erosion proceeds, the cusp moves gradually equatorward, maintaining its $\sim 4^{\circ}$ width. In Figure $5(\mathrm{c})$ the onset of a strong northward IMF facilitates reconnection with field lines near the cusp poleward boundary, resulting in a deeper penetration of magnetosheath plasma and a wider cusp. EFFICIENCY OF EROSION OF DAYSIDE MAGNETIC FLUX

Having established in Figure 4 that the flux in the polar cap depends chiefly on the amount of southward interplanetary magnetic flux 
impingent on the magnetosphere in the previous 45-minutes, and that the cusp is very nearly stationary for all values of a northward-directed IMF, we can use the observations for negative $B_{Z}$ to determine the efficiency of erosion of dayside flux in the context of the model of Coroniti and Kenne1 (1971). For this purpose, we have assumed a quiescent polar cusp equatorward boundary at $\Lambda=76.5^{\circ}$ for all $\mathrm{B}_{Z} \geq 0$. By then calculating an average value of $\mathrm{B}_{-2}$ for the 45 minutes prior to each polar cusp observation, and multiplying this average southward component by either the measured solar-wind velocity (when available) or by a typical value of $450 \mathrm{~km} / \mathrm{s}$, a value for the total southward flux brought to the front of the magnetosphere was obtained. Since the polar cusp observations were restricted to $9-15$ hrs. MLT, we have assumed a region of interaction between the IMF and the magnetosphere $22 \mathrm{R}_{\mathrm{E}}$ wide in the GSM equatorial plane. This estimate is based on the magnetic meridian map of Fairfield (1968). The eroded flux was then estimated for each cusp observation which followed a 45-minute period in which some southward flux was applied to the magnetosphere. The eroded flux was calculated by assuming a dipole field near the earth and integrating the total flux crossing the surface of the earth between 9 and 15 hrs. MLT and between the quiescent polar cusp equatorward boundary $\left(76.5^{\circ}\right)$ and the observed boundary. Values of $\Phi$ applied and $\Phi$ eroded are plotted in Figure 6 . The best linear fit to the data is shown by the solid line, which corresponds to a ratio $\Phi$ eroded $/ \Phi$ applied of 0.092 , or a $9.2 \%$ erosion efficiency. Dashed lines for $20 \%$ and $30 \%$ efficiencies are shown for comparison. 
As shown in Figure 3, the amount of flux added to the polar cap at equilibrium is expected to be roughly one-half the value which would have been added to the polar cap if convection did not return some flux to the dayside. That is, $\Phi_{P C}-\Phi_{P C}(0)=\dot{\Phi}_{0} \Delta$ at equilibrium, while the total flux added to the polar cap in time $2 \Delta$, assuming no convection, would be $2 \dot{\Phi}_{0} \Delta$. Therefore, one would expect an actual merging efficiency of approximately twice the value of the equilibrium erosion efficiency including convection. The resulting value of $\sim 18 \%$ is in good agreement with the predictions of Levy, Petschek and Siscoe (1964) and with the known ratio of about 0.20 between the solar-wind electric potential across the magnetosphere $(\sim 250 \mathrm{kv})$ and the magnetospheric cross-tail potential $(\sim 50 \mathrm{kv})$.

\section{SUMMARY}

By considering only those periods when the delay time from the interplanetary observing position to the magnetosphere is less than $\sim 5$ minutes, it is found that, irrespective of substorm activity: (1) The 45-minute average value of interplanetary $B_{Z}$ predicts the latitudes of the poleward and equatorward boundaries of dayside cusp electron precipitation with rms errors of $1.34^{\circ}$ and $1.16^{\circ}$ respectively; (2) Both boundaries move equatorward by about $5^{\circ}$ as $B_{Z}$ varies from 0 to $-6 \gamma$, the cusp remaining about $4^{\circ}$ wide; (3) As $B_{Z}$ becomes more positive, the equatorward boundary moves only slightly more poleward ( $\frac{1}{2}^{\circ}$ between $B_{Z}=2 \gamma$ and $B_{Z}=6 \gamma$ ), while the poleward boundary moves significantly toward higher latitudes, resulting in a cusp approximately $7^{\circ}$ wide for $B_{Z}=6 \gamma ;(4)$ The amount of flux added to the polar cap is about $9.2 \%$ of the total southward flux impingent on the magnetosphere in the previous 45 minutes. 
The observed equatorward displacement of the polar cusp during periods of southward IMF serves to confirm and quantify the previous observations of Aubry et a1 (1970), Russe11 et al (1971), and Burch (1972). The observation that the cusp becomes wider as the IMF becomes more northward, and that this widening is caused by the poleward motion of the cusp high-latitude boundary while the equatorward boundary remains nearly stationary, has not previously been predicted or investigated. A possible explanation is that reconnection between the IMF and open geomagnetic field lines (which are southward near the dayside magnetopause) is facilitated when the IMF is northward, allowing deeper penetration of magnetosheath plasma into the high-latitude magnetosphere, where it is precipitated closer to the poles.

\section{ACKNOWLEDGMENTS}

I am grateful to Dr. R. A. Hoffman for providing access to his OGO-4 data and for helpful discussions. Mr. S. R. Tammara provided significant programming assistance. Interplanetary magnetic field data were provided by NSSDC. 


\section{3 \\ REFERENCES}

Akasofu, S.-I., Midday auroras at the south pole during magnetospheric substorms, J. Geophys. Res., 77, 2303, 1972.

Aubry, M. P., C. T. Russe11, and M. G. Kivelson, On inward motion of the magnetopause preceding a substorm, J. Geophys. Res., 75, 7018, 1970 .

Burch, J. L., Precipitation of low-energy electrons at high latitudes: effects of interplanetary magnetic field and dipole tilt angle, J. Geophys. Res., 77, 6696, 1972.

Coroniti, F. V., and C.F. Kennel, Magnetopause motions, DP-2 and the growth phase of magnetospheric substorms, UCLA Preprint No. PPG-99, 1971.

Fairfield, D. H., Average magnetic field configuration of the outer magnetosphere, J. Geophys. Res., 73, 7329, 1968.

Fairfield, D. H., Average and unusual positions of the earth's magnetopause and bow shock, J. Geophys. Rev., 76, 6700, 1971.

Heikkila, W. J., and J. D. Winningham, Polar cap auroral particle fluxes, EOS Trans. AGU, 53, 501, 1972 .

Hoffman, R. A., and D. S. Evans, Field aligned electron bursts at high latitudes observed by OG0-4, J. Geophys. Res., 73, 6201, 1968.

Hoffman, R. A., and J. L. Burch, Electron precipitation patterns and substorm morphology, J. Geophys. Res., in press, 1973.

Kivelson, M. G., C. T. Russe11, M. Neugebauer, F. L. Scarf and R. W. Fredricks, The dependence of the polar cusp on the north-south component of the interplanetary magnetic field, J. Geophys. Res., in press, 1973 . 
Levy, R. H., H. E. Petschek and G. L. Siscoe, Aerodynamic aspects of the magnetospheric flow AIAA Journal, 2, 2065, 1964.

Meng, C.-I., Variation of the magnetopause position with substorm activity, J. Geophys. Res. , 75, 3252, 1970.

Russe11, C. T., C. R. Chappe11, M. D. Montgomery, M. Neugebauer, and

F. L. Scarf, Ogo 5 observations of the polar cusp on November 1, 1968,

J. Geophys. Res., 76, 6743, 1971. 


\section{5}

\section{FIGURE CAPTIONS}

Figure 1. A typical example of electron precipitation within a few hours of noon MLT when the interplanetary magnetic field is northward.

Figure 2. Explorer 34 interplanetary monitoring positions for the 75 polar cusp correlations analyzed herein. Bow shock and magnetopause locations are from Fairfield (1971).

Fi.gure 3. Schematic representation of the interplanetary $B_{z}$ and the resulting amount of magnetic flux added to the polar cap, assuming constant solar-wind velocity, according to the model of Coroniti and Kenne1 (1971). In all three cases the average value of $B_{z}$ over the time period $t_{0} \leq t \leq\left(t_{0}+2 \Delta\right)$ is $-B_{0}$.

Figure 4. Locations of the poleward and equatorward boundaries of dayside cusp electron precipitation for 45-minute $B_{z}$ averages between $-6 \gamma$ and $+6 \gamma$. Solid curves represent second-order polynomial fits to the data.

Figure 5. Schematic representations of a possible explanation for the observed dependence of polar cusp latitudinal width on $B_{Z}$. (a) For a nearly horizontal IMF reconnection is minimized and the cusp is $\sim 4^{\circ}$ wide. (b) After the onset of southward IMF, increased reconnection with closed field lines occurs, and dayside flux is eroded into the tail. The cusp remains $\sim 4^{\circ}$ wide. (c) For a strong northward IMF, increased reconnection occurs with field lines near the cusp poleward boundary, allowing magnetosheath plasma to penetrate deeper into the high-1atitude magnetosphere. 


\section{$-22=16$}

Figure 6. A plot of the observed relationships between the amount of GSM southward magnetic flux brought. to the front of the magnetosphere in a 45-minute period and the amount of dayside flux eroded into the tail. The eroded flux has been determined by calculating the amount of dipole flux crossing the earth's surface between the observed polar cusp equatorward boundary and an assumed quiescent boundary (for northward IMF) at $\Lambda=76.5^{\circ}$. The solid line, representing $\Phi_{\text {eroded }} / \Phi_{\text {applied }}=$ 0.092 , was obtained by a least squares fit to the data. 


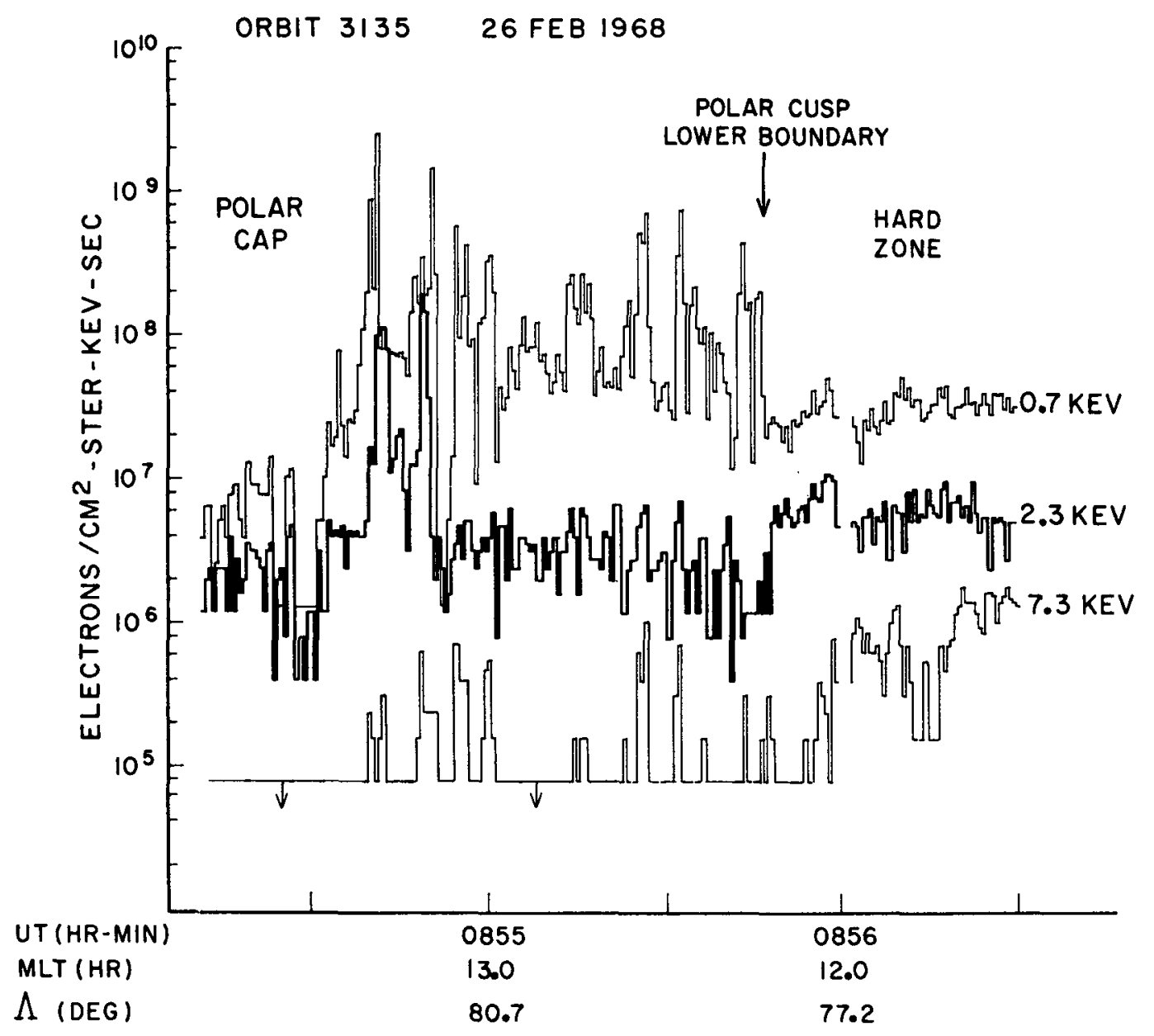




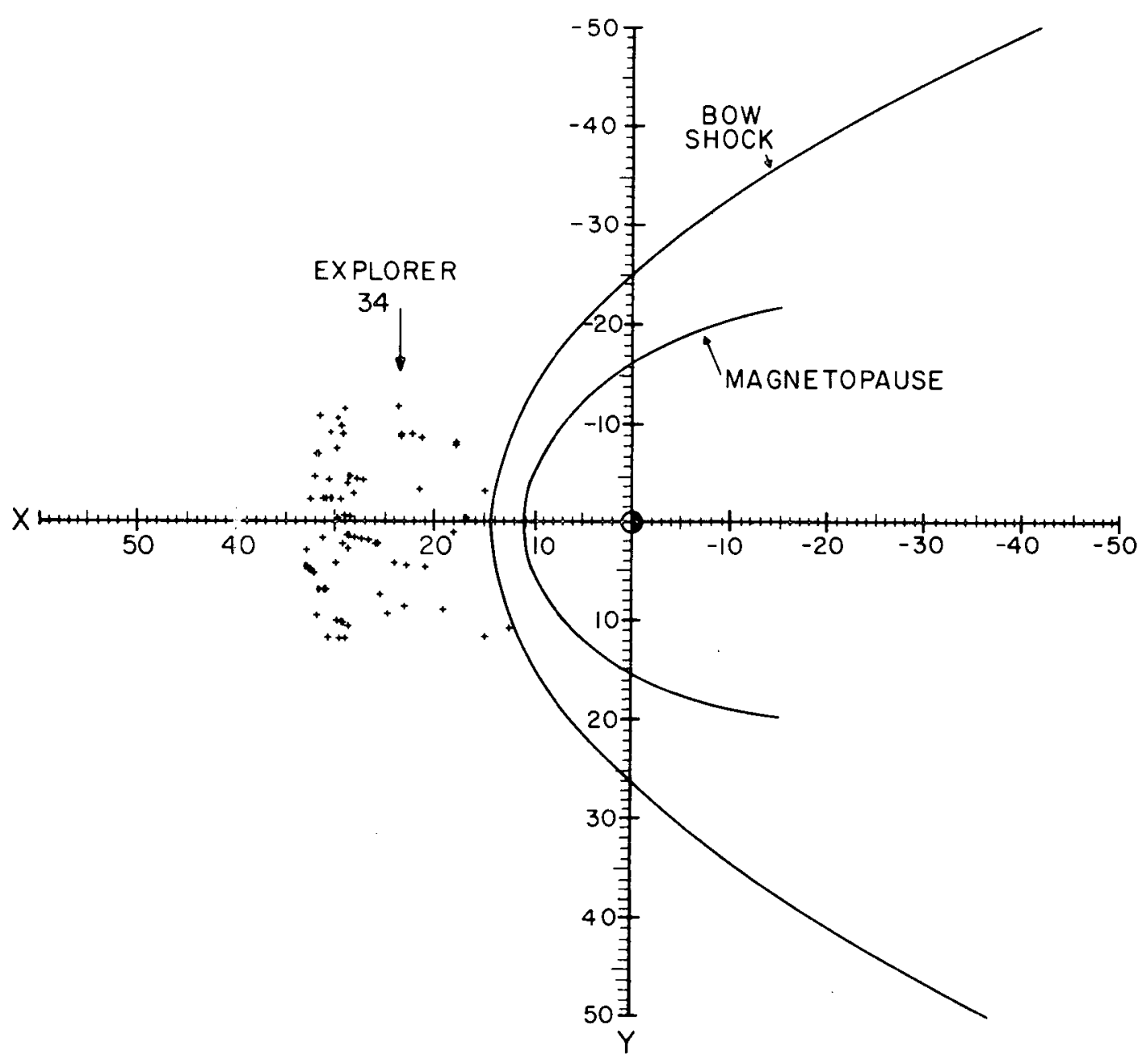

a

FIGURE 2 


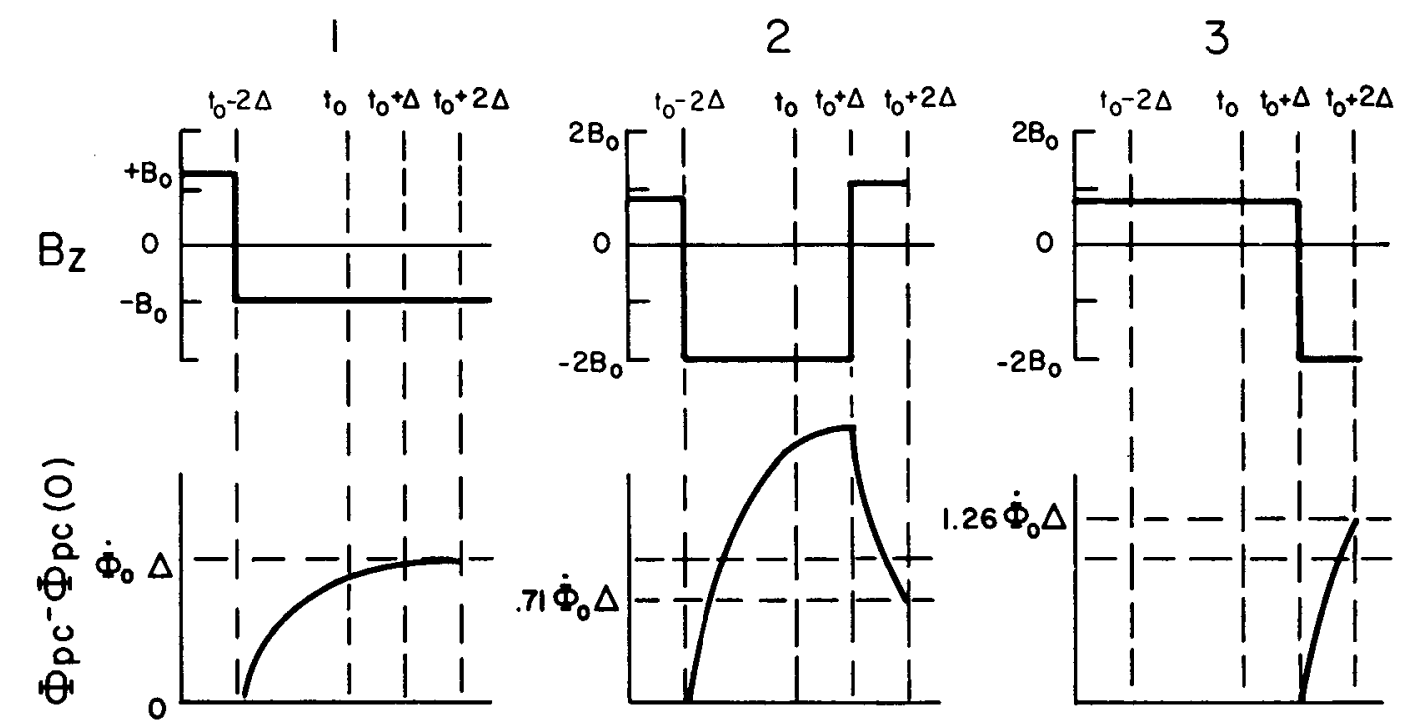

$D$

FIGURE 3 


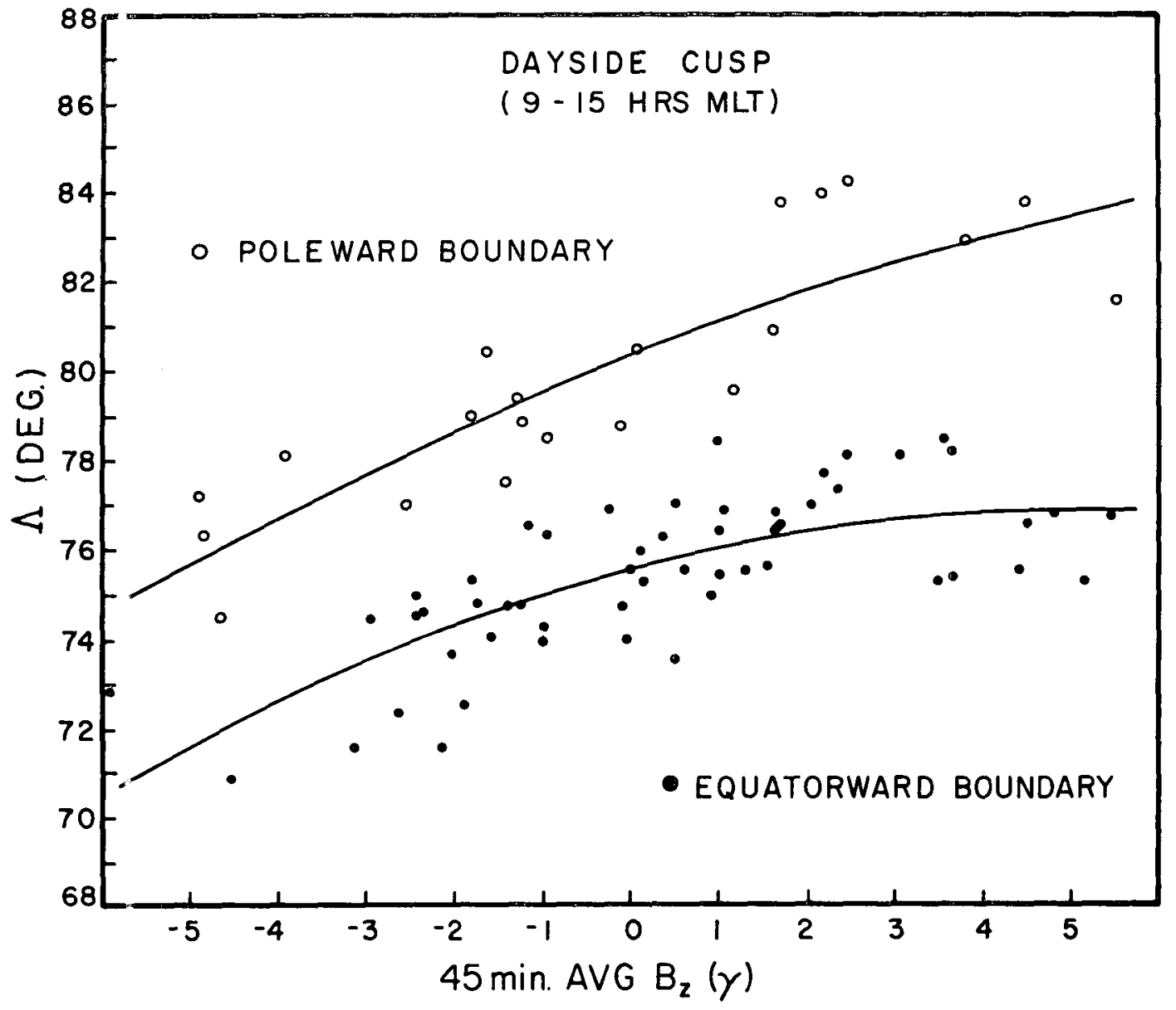

FIGURE 4 


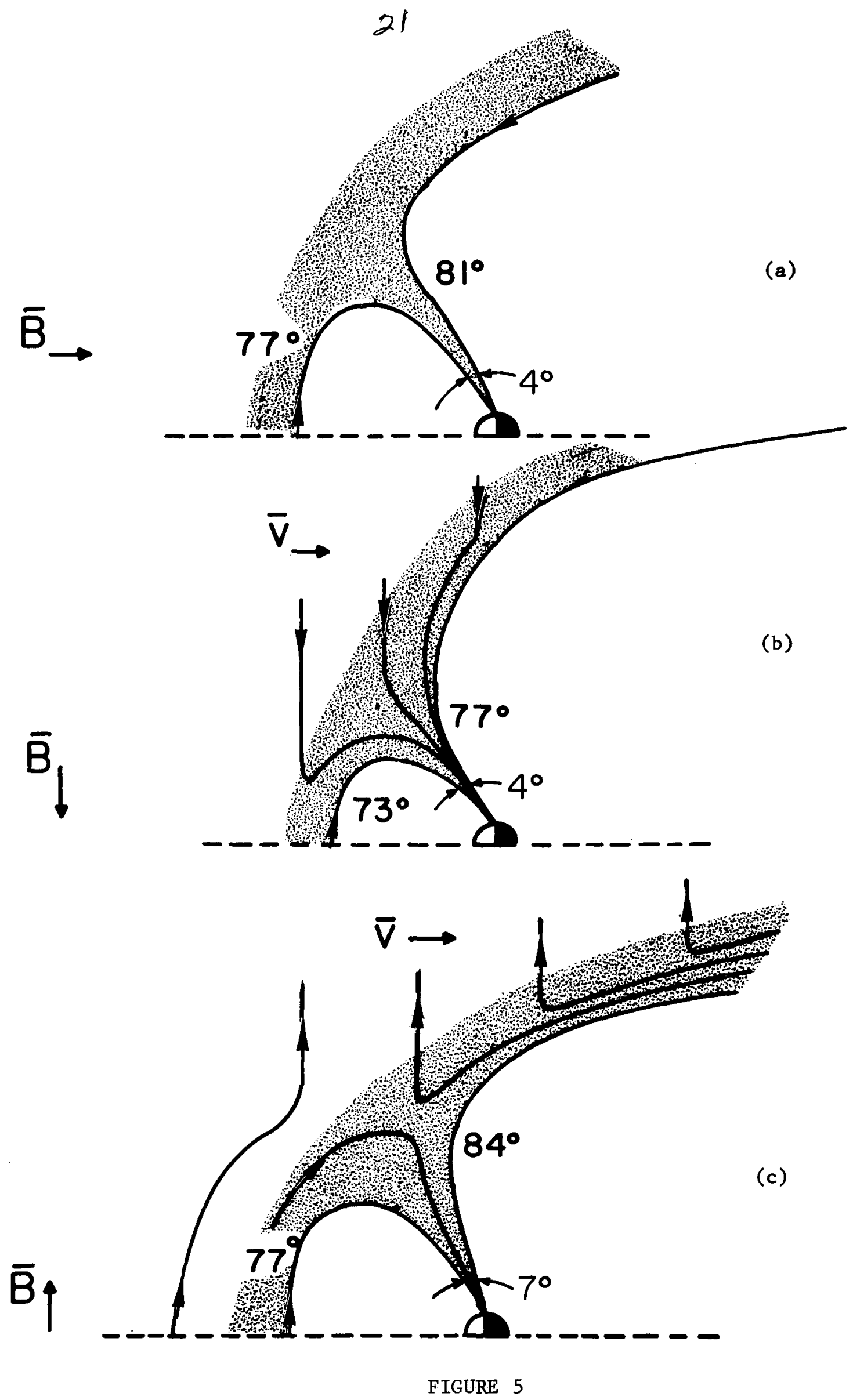




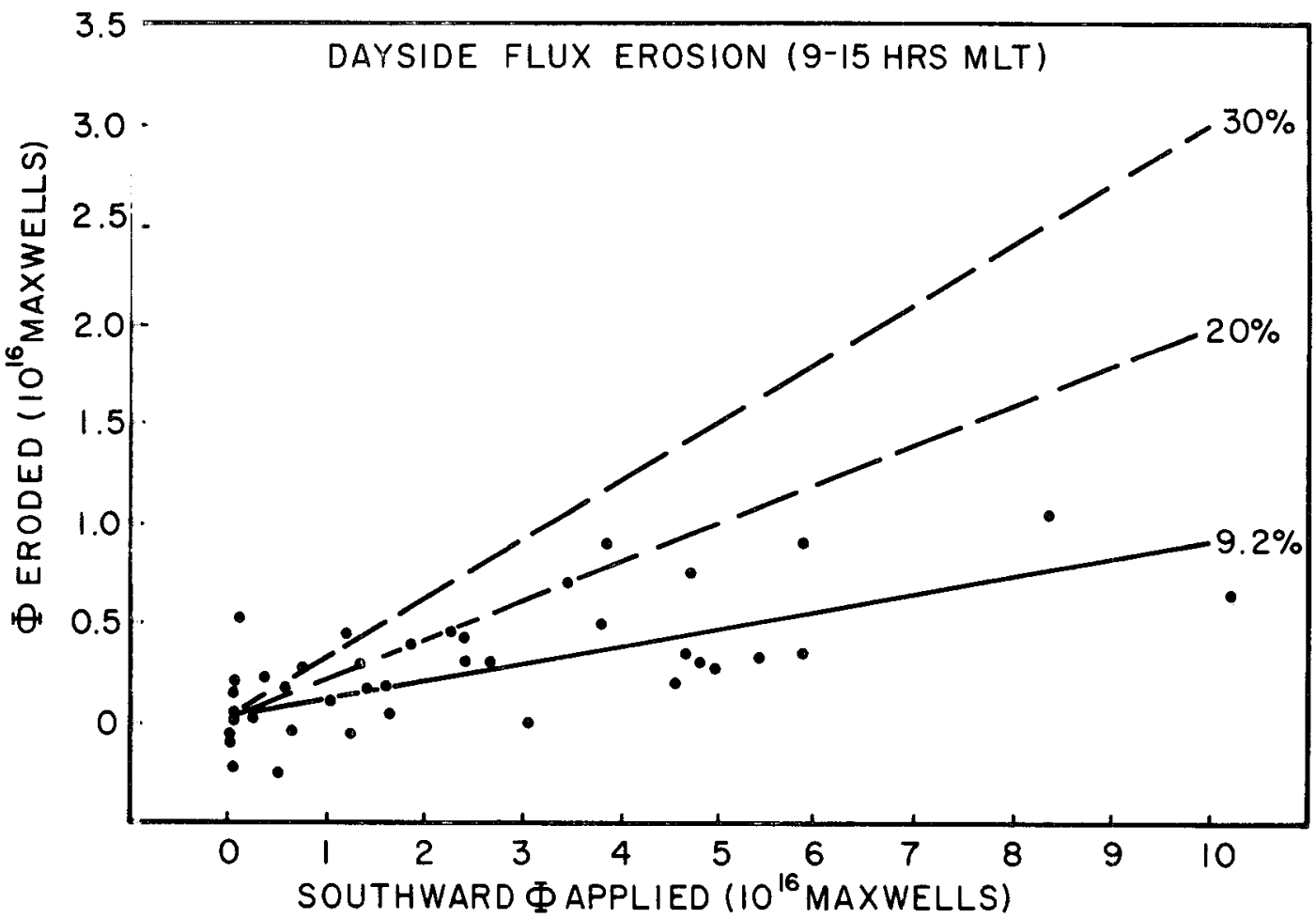

FIGURE 6 